\section{'GoldJim' Peach}

\section{John R. Clark ${ }^{\mathbf{1}}$ and Roy C. Rom ${ }^{2}$ \\ Department of Horticulture, University of Arkansas, Fayetteville, AR 72701}

Additional index words. Prunus persica, fruit breeding, bacterial spot, Xanthomonas campestris pv. pruni, processing peaches

'GoldJim' is the fourth non-melting flesh, clingstone peach [Prunus persica $($ L.) Batsch.] released by the Univ. of Arkansas peach breeding program. Previous releases were 'Allgold' and 'Goldilocks' in 1983 (Moore et al., 1984), and 'Goldnine' in 2000 (Clark et al., 2001).

'GoldJim' is a processing peach that ripens in mid-season and has exceptional processing quality and above-average resistance to bacterial spot [caused by Xanthomonas campestris pv.pruni (Smith) Dye]. It is released to complement or replace 'Goldilocks' and 'Babygold 5', both processing cling peaches with similar maturity dates. 'GoldJim' is named in honor of Univ. of Arkansas fruit breeder James N. Moore, Distinguished Professor Emeritus.

\section{Origin}

'GoldJim' resulted from a cross of Ark. 24 x NJC-70 made at the Univ. of Arkansas Fruit Substation in 1971 (Fig. 1). The seedling trees of this cross were planted at this location in 1972, and 'GoldJim' was selected in 1977 by J.N. Moore and R.C.R. and tested thereafter as Ark. 219.

The primary testing of 'GoldJim' was at the Fruit Substation, Clarksville, Ark. [westcentral Arkansas, lat. $35^{\circ} 31^{\prime} 58^{\prime} \mathrm{N}$ and long. $93^{\circ} 24^{\prime} 12^{\prime} \mathrm{W}$; U.S. Dept. of Agriculture (USDA) hardiness zone 7a; soil type Linker fine sandy loam (Typic Hapludults)]. It was also tested at the Southwest Arkansas Research and Extension Center, Hope [southwest Arkansas, lat. $33^{\circ} 42^{\prime} 30^{\prime}$ and long. $93^{\circ} 33^{\prime} 0^{\prime}$; USDA hardiness zone 8a, soil type Bowie fine sandy loam (Fragic Palendults)]. In all tests, trees were spaced $5.5 \mathrm{~m}$ apart, trained to an open-center system, pruned annually, and fertilized annually with either complete or nitrogen fertilizers. Pests were managed by using a pesticide control program common to commercial production in the area,

Received for publication 29 Sept. 2000. Accepted for publication $10 \mathrm{Feb}$. 2001. Published with approval of the Director, Arkansas Agricultural Experiment Station, manuscript no. 00088. We thank Jim Moore, Curt Rom, Bryan Blackburn, Effie Gilmore, David Gilmore, Patrick Byers, Robert Bourne, Jack Young, Stanley Brown, William Sistrunk, Alfredo Gonzalez, and Ana Lucia VasquezCaicedo for input and/or data collection during the evaluation of 'GoldJim'. The cost of publishing this paper was defrayed in part by the payment of page charges. Under postal regulations, this paper therefore must be hereby marked advertisement solely to indicate this fact.

${ }^{1}$ Professor.

${ }^{2}$ University Professor Emeritus. including applications of fungicides and insecticides. No bactericides were applied; thus, no attempt was made to control bacterial spot during evaluation. Fruit were thinned by hand as needed to a distance of $\approx 12 \mathrm{~cm}$ between fruit prior to pit hardening but after shuck split.
Trials consisting of two-tree observational plots were maintained at Clarksville and data were collected from these trees or the original tree from 1978 through 1999. Data are reported here for a maximum of 9 years for any data point. Comparison cultivars also planted at this site included 'Babygold 5', 'Babygold 7', 'Allgold', and 'Goldilocks'. Field observations included bloom date, bloom intensity, and fruit maturity date, while fruit ratings were taken for size, shape, firmness, skin color, flesh color, finish (presence of surface blemishes, cracks, or other skin surface defects), and flavor, and trees were rated for vigor, crop load, and health. The rating scale for all these variables was 1 to 10 , with a rating of 10 most desirable, with the exception of vigor, in which

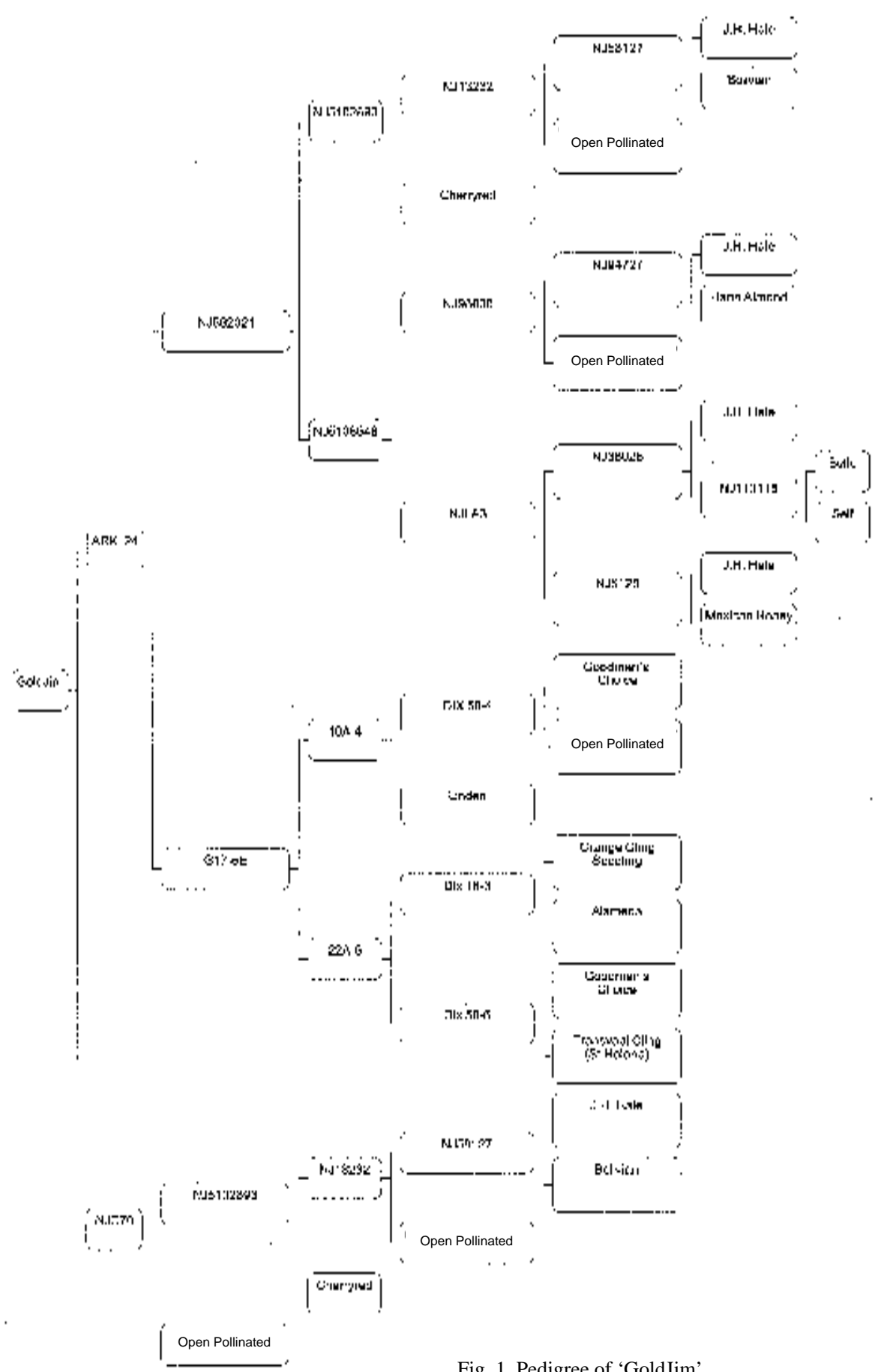

Fig. 1. Pedigree of 'GoldJim'. 
a rating of 10 indicated excessive vigor. A rating of 7 to 8 would be most desirable for vigor in this rating scale. Additionally, a fivefruit sample was collected for 'GoldJim' for 9 years (1988, 1990-95, 1997-98) and average fruit weight, size, firmness [using a model FT327 fruit pressure tester with an 11-mmdiameter probe, (McCormick Fruit Tree Co., Yakima, Wash.)], color (7 years only, measurements being taken using a Minolta model CR200 chroma meter, Minolta, Ramsey, N.J.), and soluble solids (using a bench refractometer) were determined. Not all variables were measured in some years as noted in the text and in the data tables. A replicated trial at Hope was established in 1992 and data were collected for yield and fruit weight in 1995 and 1997 (crops were lost or reduced because of spring frost in 1994 and 1996). Four singletree replications arranged in a randomized complete-block design were utilized in this planting, and data for each year were analyzed separately. Data from the replicated trial at Hope were analyzed by analysis of variance and means separated by LSD (SAS Institute, 1989).

Processing evaluations were conducted for 9 years by the Dept. of Food Science, Univ. of Arkansas, from $\approx 20-\mathrm{kg}$ samples collected at Clarksville and delivered to Fayetteville. Data are not reported for all years for all variables. For all samples processed, fruit were peeled, pitted, and blanched in live steam for $5 \mathrm{~min}$ and then packed in $0.5-\mathrm{L}$ glass jars. The jars were exhausted for $10 \mathrm{~min}$, sealed, and cooked for $25 \mathrm{~min}$ in boiling water. Soluble solids were measured with a bench refractometer from a blended, filtered sample. Titratable acidity and $\mathrm{pH}$ were measured on a 10 -g sample diluted in $50 \mathrm{~mL}$ of deionized water. Samples were titrated to a $\mathrm{pH}$ of 8.2 using $0.1 \mathrm{~N} \mathrm{NaOH}$. These measurements were done on both fresh (represented as the fresh slice data in Table 1), and processed samples (represented in the canned slices and puree data in Table 1). Processed sample color was determined by a Gardner Color Difference Meter (Colorguard System 1000 Colorimeter, Gardner/Neotec Instrument Division, Pacific Scientific Co., Silver Spring, Md.) that was standardized with a plaque $(\mathrm{L}=93.5, \mathrm{a}=1.4$, and $\mathrm{b}=3.2)$. In some years, sensory evaluations were conducted on samples 3 to 4 months after processing by a trained panel of 10 to 15 evaluators, who rated the samples for color and flavor.

\section{Description and performance}

Fruit of 'GoldJim' ripen an average of $17 \mathrm{~d}$ after 'Allgold', with 'Goldilocks', 2 d before 'Babygold 5', and $10 \mathrm{~d}$ before 'Babygold 7' (Table 2). Yield of 'GoldJim' was significantly higher than that of 'Babygold 7' in both years, and higher than that of 'Babygold 5' in 1997 (Table 3). 'GoldJim' also had higher initial fruit set that did 'Babygold 7' and 'Babygold 5' (data not shown). This provides increased reliability in cropping and offsets some risk of spring frost damage because of slightly earlier bloom than most comparable cultivars.
Table 1. Quality characteristics of processed samples of processing peach cultivars grown at the Univ. of Arkansas Fruit Substation, Clarksville.

\begin{tabular}{|c|c|c|c|c|}
\hline & GoldJim & Allgold & Goldilocks & Babygold 5 \\
\hline \multicolumn{5}{|c|}{ Fresh slices $^{2}$} \\
\hline Soluble solids (\%) & 12.3 & 11.1 & 11.7 & 12.7 \\
\hline $\mathrm{pH}$ & 3.87 & 3.74 & 3.83 & 3.82 \\
\hline Titratable acidity ${ }^{y}$ & 3.95 & 4.29 & 3.88 & 4.22 \\
\hline \multicolumn{5}{|l|}{ Color $^{x}$} \\
\hline $\mathrm{L}$ & 59.0 & 61.7 & 60.7 & 62.2 \\
\hline $\mathrm{a}$ & 18.6 & 13.5 & 18.5 & 16.5 \\
\hline $\mathrm{b}$ & 32.5 & 32.9 & 34.1 & 34.1 \\
\hline \multicolumn{5}{|c|}{ Canned slices ${ }^{w}$} \\
\hline Soluble solids (\%) & 14.0 & 12.9 & 14.0 & 13.7 \\
\hline $\mathrm{pH}$ & 3.85 & 3.74 & 3.90 & 3.80 \\
\hline Titratable acidity & 3.64 & 3.92 & 2.56 & 4.30 \\
\hline \multicolumn{5}{|l|}{ Color } \\
\hline $\mathrm{L}$ & 66.8 & 67.9 & 66.6 & 67.9 \\
\hline $\mathrm{a}$ & 13.6 & 11.0 & 13.3 & 9.4 \\
\hline b & 36.8 & 38.5 & 37.6 & 38.7 \\
\hline \multicolumn{5}{|c|}{ Peach puree ${ }^{w}$} \\
\hline Soluble solids (\%) & 12.7 & 11.1 & 12.2 & --- \\
\hline $\mathrm{pH}$ & 3.81 & 3.73 & 3.82 & --- \\
\hline Titratable acidity & 3.57 & 3.66 & 3.09 & --- \\
\hline \multicolumn{5}{|l|}{ Color } \\
\hline $\mathrm{L}$ & 64.6 & 64.5 & 63.5 & --- \\
\hline $\mathrm{a}$ & 11.6 & 7.2 & 11.6 & --- \\
\hline $\mathrm{b}$ & 34.9 & 35.1 & 35.5 & --- \\
\hline
\end{tabular}

${ }^{\mathrm{z}}$ Means for 4 years.

y Titratable acidity as malic acid in $\mathrm{g} \cdot 100^{-1} \mathrm{~mL}$.

${ }^{\mathrm{x}}$ Color as determined by color difference meter; the lower the $\mathrm{L}$ value, the darker the sample; the higher the a value, the redder the sample; the higher the $b$ value, the more yellow the sample.

weans for 3 years.

Table 2. First harvest and bloom dates and subjective evaluations (based on a 1-10 scale, with a rating of 6 or below considered unacceptable) of fruit and tree characteristics of processing peach cultivars grown at the Univ. of Arkansas Fruit Substation, Clarksville. Means for 7 years, with the exception of bloom dates, which are means of 9 years.

\begin{tabular}{lccccc}
\hline \hline & GoldJim & Allgold & Goldilocks & Babygold 5 & Babygold 7 \\
\hline Fruit & & & & & \\
$\quad$ First harvest date & 19 July & 2 July & 19 July & 21 July & 29 July \\
Size $^{\mathrm{z}}$ & 7.1 & 9.0 & 7.7 & 7.7 & 8.8 \\
Shape $^{\mathrm{y}}$ & 7.7 & 7.6 & 7.7 & 7.6 & 6.4 \\
Firmness $^{\mathrm{x}}$ & 9.0 & 7.8 & 8.1 & 7.7 & 8.6 \\
Skin color $^{\mathrm{w}}$ & 8.6 & 8.1 & 8.0 & 7.3 & 7.6 \\
Flesh color $^{\mathrm{v}}$ & 8.4 & 7.6 & 7.1 & 6.4 & 6.4 \\
Finish $^{\mathrm{u}}$ & 8.4 & 8.0 & 7.7 & 6.7 & 7.4 \\
Flavor $^{\mathrm{t}}$ & 8.3 & 7.4 & 7.6 & 7.3 & 6.8 \\
Plant & & & & & \\
Bloom date in March & & & & & \\
$\quad 10 \%$ & 13 & 15 & 17 & 17 & 15 \\
$\quad$ Full $^{\text {Vigor }}$ & 18 & 23 & 24 & 24 & 23 \\
Crop & 7.4 & 8.3 & 8.1 & --- & -- \\
Health $^{\mathrm{q}}$ & 9.0 & 8.6 & 8.3 & -- & -- \\
\hline
\end{tabular}

${ }^{\mathrm{z}} 10=$ very large.

${ }^{\mathrm{y}} 10=$ round or mostly round, or uniform and free of bulging suture and blossom-end tip.

${ }^{x}$ Based on hand pressure applied to intact, unpeeled fruit, with $10=$ very firm fruit.

"Preferred is a light, bright-red, over-orange ground color, with $10=$ full expression of these colors.

${ }^{\vee}$ Preferred is uniform orange color with no trace of red pigment, with $10=$ no red in flesh and uniform orange color.

"Preferred is bright finish free of any blemish or skin cracks or inconsistencies, with $10=$ free of all surface blemishes or skin cracks.

'Based on fresh samples. Higher values indicate good sweetness and expression of peach flavor and low astringency or other undesirable flavor components.

'Based on good but not excessive shoot growth; optimum rating 7 to 8 with 10 being excessive.

${ }^{\mathrm{r}} 10=$ full crop; $9=90 \%$ crop, etc.

${ }^{\mathrm{q}} 10=$ no disease or health concerns. The most important disease in this rating is bacterial spot, a higher rating indicating a lower occurrence of this disease. Non-disease factors affecting health could include mineral deficiencies or insect damage. 
Table 3. Yield and fruit weight of processing peach cultivars grown at the Southwest Research and Extension Center, Hope, Ark. ${ }^{2}$

\begin{tabular}{lcc}
\hline Cultivar & Yield/tree (kg) & Fruit wt (g) \\
\hline & 1995 & \\
GoldJim & $64.5 \mathrm{a}$ & $132 \mathrm{~b}$ \\
Babygold 5 & $50.8 \mathrm{ab}$ & $189 \mathrm{a}$ \\
Babygold 7 & $43.3 \mathrm{~b}$ & $208 \mathrm{a}$ \\
& 1997 & \\
GoldJim & $98.8 \mathrm{a}$ & $149 \mathrm{~b}$ \\
Babygold 5 & $51.1 \mathrm{~b}$ & $247 \mathrm{a}$ \\
Babygold 7 & $68.6 \mathrm{~b}$ & --- \\
\hline${ }^{2}$ Planting established in 1992. &
\end{tabular}

${ }^{\mathrm{y}}$ Mean separation within columns and years by LSD, $P \leq 0.05$.

The average size rating for 'GoldJim' was 7.1 on a 10 -point scale (where $10=$ very large fruit) (Table 2). Average fruit weight for 'GoldJim' over 9 years was $183 \mathrm{~g}$, as compared to $205 \mathrm{~g}$ for 'Allgold', $150 \mathrm{~g}$ for 'Goldilocks', and 166 and $180 \mathrm{~g}$ for 'Babygold 5' and 'Babygold 7', respectively (data not shown). Among 9 years of data collection, average fruit weight ranged from the heaviest of $285 \mathrm{~g}$ in 1998 to $132 \mathrm{~g}$ in 1995 (data not shown). At Hope, fruit of 'GoldJim' were smaller than those of the Babygold cultivars (Table 3), possibly because of its heavier crop load. Close attention should be paid to proper fruit thinning in the production of 'GoldJim' because heavy bloom and fruit set can lead to smaller than optimal fruit size.

'GoldJim' fruit are generally round, averaging $6.4 \mathrm{~cm}$ in length and $7.0 \mathrm{~cm}$ in diameter. Fruit shape ratings resembled those of other cultivars (Table 2). 'GoldJim' was firmer that the comparison cultivars (Table 2) and had a 6year average firmness of $6.6 \mathrm{~kg}$ as compared to $3.2 \mathrm{~kg}$ for 'Allgold' and $4.5 \mathrm{~kg}$ for 'Goldilocks' (data not shown). Skin color of 'GoldJim' was rated as attractive or better than the other cultivars (Table 2). Skin surface averaged $45 \%$ blush over 7 years, with skin ground color values of 69.5 for $L$ (the lower the $L$ value, the darker the sample), 17.3 for a (the higher the a value, the redder the sample), and 52.9 for $b$ (the higher the $\mathrm{b}$ value, the more yellow the sample). Skin over color values were $\mathrm{L}=39.8$, $a=37.0$, and $b=21.3$. Likewise, fruit finish ratings (reflecting a lack of skin defects, such as cracking, bacterial spot lesions, etc.) were very good for 'GoldJim' (Table 2). Flesh color ratings for 'GoldJim' exceeded those of the other evaluated cultivars, which reflects its orange-gold flesh color with lack of red pigment in the flesh, a highly desirable characteristic for a processing peach (Table 2). 'GoldJim' has consistently been among the highest-rated genotypes for flesh color in the Arkansas breeding program. No red pigment was observed in the flesh of 'Gold Jim' in any years, whereas in most genotypes red pigment is often observed.
'GoldJim' had better flavor than the comparison cultivars (Table 2) and soluble solids content (12.4\%) similar to the other Arkansas cultivars (data not shown). In most years, split pits were rare in 'GoldJim'; and when they were observed, they affected fewer fruits than was true of other processing peach cultivars (data not shown). Additionally, fruit of 'GoldJim' have been observed to hang well on the tree at maturity without loss of quality, which facilitates harvesting and may reduce the number of harvests required.

Analyses conducted by the Dept. of Food Science indicated that 'GoldJim' consistently had exceptional processed quality. Soluble solids content of 'GoldJim' exceeded that of 'Allgold' and 'Goldilocks' for fresh, canned, or pureed samples, and was surpassed only by 'Babygold 5' for fresh slices (Table 1). Fruit $\mathrm{pH}$ and titratable acidity values were generally similar among the cultivars evaluated, whether measured on fresh, canned, or pureed samples (Table 1). Minor differences were apparent among L values, and the 'GoldJim' value for fresh slices was slightly lower than that of 'Allgold', and to some extent lower than that of 'Babygold 5', possibly reflecting the more orange color of the flesh. However, processed samples indicated similar L values among cultivars. The a values for 'GoldJim' were usually higher than for 'Allgold' but similar to that of 'Goldilocks'. Values for b were comparable among all cultivars. In 9 years of sensory evaluations on the pureed samples, color of 'GoldJim' was rated higher than that of 'Allgold' and comparable to 'Goldilocks', while flavor of 'GoldJim' was rated higher than that of both these cultivars. In a more detailed study comparing sensory evaluations of canned slices of 11 genotypes ('Allgold', 'Goldilocks', 'Babygold 5', 'Babygold 7', 'Babygold 8', and five breeding selections), 'GoldJim' was the highest rated for sweet and "peachy" flavor attributes (Vasquez-Caicedo, 1999).

Average $10 \%$ bloom date at Clarksville for 'GoldJim' was 13 Mar., 2 to $4 \mathrm{~d}$ earlier than that of the comparison cultivars, and average date of full bloom was 18 Mar., 5 to 6 d earlier than that of the other cultivars (Table 2). Full bloom date varied from 7 to 30 Mar. over 9 years. Bloom dates at Hope indicated that in 1995 'GoldJim' bloomed on the same date as 'Babygold 7' and $1 \mathrm{~d}$ earlier than 'Babygold 5', whereas in 1997 it bloomed 6 and $8 \mathrm{~d}$ earlier than these two cultivars, respectively (data not shown). Bloom density was greater in 'GoldJim' than in 'Allgold', 'Goldilocks', 'Babygold 5', and 'Babygold 7' (average for 9 years, data not shown). Flowers of 'GoldJim' are non-showy and self-fertile. Leaf glands are globose. Tree vigor was rated slightly lower for 'GoldJim' than for 'Allgold' and 'Goldilocks' (Table 2), although this differ- ence was minimal and would not affect canopy management decisions. Crop load ratings taken at fruit maturity were slightly higher for 'GoldJim' than for these same cultivars (Table 3). Consistent cropping was achieved in all years of fruiting except for years when spring frosts eliminated the crop. Ratings for tree health for 'GoldJim' were intermediate to those for 'Allgold' and 'Goldilocks' (Table 2). 'GoldJim' was comparable to 'Allgold' in bacterial spot resistance, and less susceptible to this disease than were 'Goldilocks' and 'Babygold 5' (data not shown). In some years a limited number of bacterial spot lesions were seen on leaves of 'GoldJim', but defoliation was not a concern. Only on rare occasions were bacterial spot lesions seen on fruit and these were very small. No other disease problems have been seen on 'GoldJim', although a commercial fungicide program was used that controlled brown rot [caused by Monilinia fructicola (G. Wint.) Honey]. Peach scab (caused by Cladosporium carpophilum Thuem.) was observed on the fruit of 'GoldJim' one year at Hope, but was less severe than on other cultivars in the planting (data not shown).

Bud hardiness of 'GoldJim' is good. In evaluations of flower buds at Clarksville following a Jan. 1986 minimum of $-24^{\circ} \mathrm{C}, 71 \%$ of 'GoldJim' flower buds survived, compared with $33 \%$ for 'Allgold' and $19 \%$ for 'Goldilocks'. Following $-22{ }^{\circ} \mathrm{C}$ on 24 and 25 Dec. 1989 at Clarksville, $40 \%$ of 'GoldJim' flower buds survived vs. $10 \%$ for 'Allgold' and $24 \%$ for 'Goldilocks'. Chilling requirement of 'GoldJim' is estimated at $\approx 750 \mathrm{~h}$ below $7{ }^{\circ} \mathrm{C}$, based on observations of budbreak and bloom in plantings with standard cultivars.

The outstanding characteristics of 'GoldJim' are its exceptional fruit quality, particularly color, flavor and firmness, consistent production, and above-average bacterial spot resistance. 'GoldJim' is recommended for trial where other processing clingstone peaches developed in eastern North America are grown.

\section{Availability}

'GoldJim' budwood is available from: J.R. Clark, 316 Plant Science, Dept. of Horticulture, Univ. of Arkansas, Fayetteville, AR 72701.

\section{Literature Cited}

Clark, J.R., J.N. Moore, and R.C. Rom. 2001 'Goldnine' peach. HortScience 36:808-810.

Moore, J.N., R.C. Rom, S.A. Brown, and W.A Sistrunk. 1984. 'Allgold' and 'Goldilocks' peaches. HortScience 19:891-892.

SAS Institute. 1989. SAS/STAT user's guide, vers. 6 , vol. 2. SAS Inst., Cary, N.C.

Vasquez-Caicedo, A.L. 1999. Effects of processing methodology on sensory flavor profiles of peaches. MS Thesis, Dept. of Food Science, Univ. of Arkansas, Fayetteville. 